\title{
DYNAMICS OF STAR CLUSTERS
}

\section{JEREMY GOODMAN and PIET HUT (EDS.)}

The enigma of core collapse receives much attention in this volume. In addition, several observational papers summarize recent techniques and results and discuss the stellar dynamical implications of the enormous progress in the quality of surface photometry, proper motion studies, radial velocity determinations, as well as space-based measurements in a variety of wavelengths. The value of these Proceedings as a standard reference work is enhanced by the inclusion of two appendices, featuring English translations of two seminal papers on stellar dynamics published in Russian and not previously available in a Western language. A third appendix contains an up-to-date catalogue of observationally determined parameters of galactic globular clusters, as well as theoretically inferred parameters. This catalogue will prove to be an essential reference for phenomenonological studies and an ideal testing ground for new theoretical developments.

\section{Audience}

The book will be of primary interest to astrophysicists. It may also, however be of value to physicists involved in the areas of statistical, plasma and mathematical physics. The volume is suitable for graduate-level supplementary reading in all astronomy programmes. 